\title{
4
}

\section{Modeling and Analysis Methods for Multi-agent Systems}

\author{
Jose R. Celaya ${ }^{1}$ and Alan A. Desrochers ${ }^{2}$ \\ ${ }^{1}$ Decision Sciences and Engineering Systems Department \\ 2Electrical, Computer, and Systems Engineering Department \\ Rensselaer Polytechnic InstituteTroy, NY 12180
}

USA

\section{Introduction}

Multi-agent systems have been studied for the past few decades. At this point in time, several multi-agent systems frameworks have been defined in order to apply the multiagent system concept to different applications. In a multi-agent system, several agents communicate and interact in order to solve a complex problem. A multi-agent system can be studied as a computer system that is concurrent, asynchronous, stochastic and distributed. These characteristics of multi-agent systems make them also a discrete-event dynamic system, and these have been studied under several analytical methodologies, particularly Petri nets. Petri nets have a well-defined mathematical structure that can be leveraged to provide formal analysis of discrete-event dynamic systems. From the discrete-event dynamic system point of view, multi-agent systems lack analysis and design methodologies. This chapter is concerned with the development of analytical methods for modeling and analysis of multi-agent systems, as well as the definition and assessment of system properties. The study of system properties is becoming more important due to the fact that we are faced more and more with handling large complex dynamic systems. Computer simulation is generally used to assess system properties and to verify that the system is achieving its design objectives. An important challenge in this field is the development of analytical methods to assess key properties of such systems. Such methods could be used to provide a preliminary analysis of the multi-agent system, providing design and operation feedback before the development of expensive simulation models. Furthermore, they will provide insight into design methodologies for multi-agent systems that will ensure that the system design under such methodologies complies with the required properties, hence being a dependable multi-agent system.

The communication and interaction among agents is critical for the overall multi-agent system design and for the proper functioning of the system. Several interaction frameworks have been defined and they range from collaboration among agents, through competition for resources requiring some level of negotiation in the multi-agent system. This work focuses particularly at the interaction level, and studies the interactions between different agents in the system.

The mission of the chapter is to present a methodology for modeling and analysis of multiagent systems at the agent interaction level. This will be carried out by studying the discrete- 
event characteristics of the interactions and using the Petri net methodologies as the modeling and analysis tool. Properties known to be important in the discrete event systems and Petri net domains will be used to study multi-agent systems. Here, properties like boundedness and liveness will be analyzed and demonstrated for multiagent systems, as they relate to deadlock avoidance in the Petri net domain. Furthermore, these properties will be related to characteristics of the interaction mechanism. If modeled properly, a deadlock found in a Petri net domain will mean that the interaction mechanism in use in the multiagent system is prone to deadlocks in the interaction among agents.

In particular, this chapter will include methodologies for mapping multi-agent systems into Petri net models. These methodologies will present the right level of detail/abstraction in order to map all the important behaviors of the interaction framework into the resulting Petri net models. Petri net analysis methods such as the reachability graph and the analysis of the network invariants will be used for the assessment of properties. Furthermore, Petri net synthesis techniques will allow the ability to provide more or less detail to the models, giving the ability to add detail/abstraction to the behavior of the multi-agent system to be modeled.

\subsection{Methodology}

The problem addressed in this chapter can be considered in two parts: properties, and methodologies for modeling and analysis. The following is the methodology followed for this work.

Properties: If a multi-agent system is regarded as a discrete-event system and modeled using Petri nets, then properties known to be important in the discrete-event systems and Petri net domains could be used to study multi-agent systems. If we consider models of multi-agent systems as discrete-event systems, an important question to consider is which properties in the Petri net domain are important? As a starting point there are properties we would like to analyze and demonstrate for multi-agent systems. Examples of these are boundedness and liveness as related to deadlock avoidance in the Petri net domain. Other properties exist that are related to performance evaluation. These properties from the Petri net domain could be related to characteristics of the communication and interaction of the multi-agent system. If modeled properly, a deadlock found in a Petri net domain will mean that the interaction mechanism in use in the multi-agent system is prone to deadlocks in the interaction among individual agents.

Methodologies for modeling and analysis: Considering that a multi-agent system can be regarded as a discrete-event system, Petri nets can be used as a modeling tool. This will require methodologies for mapping multi-agent systems into Petri net models. These methodologies will require the right level of detail/abstraction in order to map all the important behaviors of the communication and interaction framework into the resulting Petri net models. Having Petri net models of multi-agent systems will allow us to use the existing analysis methodologies for Petri nets. Important properties of discrete-event systems could be obtained with Petri net analysis methods such as the reachability graph and the analysis of the network invariants. New analysis techniques related directly to the multi-agent system domain can be designed or tailored from existing Petri net techniques. Furthermore, Petri net synthesis techniques allow us to provide more or less detail to our models giving the ability to add detail/abstraction to the behavior of the multi-agent system that we would like to model. 


\subsection{Related work}

Petri nets and Petri net extension methodologies have been used to model systems with more than one agent. Murata et al. [1] presented an algorithm to construct predicate/ transition models of robotic operations. Basically, robot actions were considered as firing transitions and the model was used for the planning of concurrent activities of multiple robots (agents). Even though the robotic system considered does not have direct interaction between the agents, the model used shows the ability of the Petri net-like models to capture interactions between the agents that are not evident in the design process. In a similar way, $\mathrm{Xu}$ et al. [2] proposed a methodology based on predicate/transition nets for multiple agents under static planning of activities. In addition, they proposed a validation algorithm for plans with parallel activities. The verification is done based on reachability graphs due to the fact that agents actions are modeled as transitions. Petri nets also have been used to model specific multi-agent system frameworks but the resulting models have not been used to provide a study of the properties of the multi-agent system. Ahn et al. [3] proposed a multi-agent system architecture for distributed and collaborative supply-chain management. The suggested architecture is aimed at discovering the structure of the supply-chain and predicting future demands based on local information sharing among the agents. A Petri net model is presented but no structural analysis of the model and no verification of the coordination activities were performed. The advantages of having a Petri net model were not exploited. The work of Leitao et al. [4], proposed a Petri net model approach to formal specification of holonic control systems for manufacturing. They developed a Petri net submodel for each of the four types of holons (agents) suggested in the ADACOR (Adaptive Holonic Control Architecture for Distributed Manufacturing Systems) architecture. There was no attempt to study the structural properties of the Petri net model in order to assess some sort of dependability in the proposed architecture. Formal modeling and specification of the multi-agent systems interaction framework has only been attempted in the holonic manufacturing system considering the contract net protocol as the interaction framework. The work presented by Hsieh in [5] proposed a new model called a collaborative Petri net and addressed the question of deadlock and undesirable state avoidance under the contract net protocol. Finally, multi-agent system survivability ${ }^{1}$ and fault tolerance using Petri net models have been used in the mobile-agent area. Lyu et al. [6] used a Stochastic Petri net model to assess survivability and fault tolerance of mobile agents systems. They use the model for design and verification of their proposed agent architecture.

\section{Introduction to Petri nets}

Petri nets are a graphical and mathematical modeling tool used to describe and analyze different kinds of real systems. Petri nets were first introduced by Carl Adam Petri in 1962 in Germany [8], and evolved as a suitable tool for the study of systems that are concurrent, asynchronous, distributed, parallel and/or stochastic. Performance evaluation has been a very successful application area of Petri nets. In addition, Petri nets have been successfully used in several areas for the modeling and analysis of distributed-software systems, distributed-database systems, flexible manufacturing systems, concurrent and parallel programs and discrete-event dynamic systems (DEDS) to mention just a few [9][8][10][11]. A

\footnotetext{
1 Survivability is the agent's ability to recognize, resist and recover from attacks [7].
} 
multi-agent system is a kind of DEDS that is concurrent, asynchronous, stochastic and distributed. From the DEDS point of view, multi-agent systems lack analysis and design methodologies. Petri net methods are used in this work to develop analytical methodologies for multi-agent systems.

Petri nets are often used in the modeling and analysis of DEDS. They include explicit conditions under which an event can occur; capturing also the relations between concurrent and asynchronous events. As a result, Petri nets are suitable for studying complex and general DEDS [10][11].

This section presents an introduction to Petri nets. Petri nets are defined followed by important properties and analysis methodologies. Finally, an example of a manufacturing application is presented.

\subsection{Petri nets definition}

Definition 1 The following is the formal definition of a Petri net [9][8][12][13]. A Petri net is a fivetuple

$$
\left(P, T, A, W, M_{0}\right)
$$

where:

$P$ is a finite set of places

$T$ is a finite set of transitions

$A \subseteq(P \times T) \cup(T \times P)$ is a set of arcs

$W: A \rightarrow\{1,2,3, \ldots\}$ is a weight function

$M_{0}: P \rightarrow Z_{+}$is the initial marking

The meanings of places and transitions in Petri nets depend directly on the modeling approach. When modeling, several interpretations can be assigned to places and transitions. For a DEDS a transition is regarded as an event and the places are interpreted as a condition for an event to occur.

Table 1 presents several typical interpretations for transitions and places. A simple Petri net example is presented in figure 1. This example is used later to define additional Petri net characteristics.

\begin{tabular}{|l|l|l|}
\hline Input Place & Transitions & Output Places \\
\hline Preconditions & Event & Postconditions \\
Input data & Computation Step & Output data \\
Input signal & Signal processor & Output signal \\
Resources needed & Task or job & Resource released \\
Conditions & Clause in logic & Conclusion \\
Buffers & Processor & Buffer \\
\hline
\end{tabular}

Table 1. Modeling interpretations of transitions and places [8].

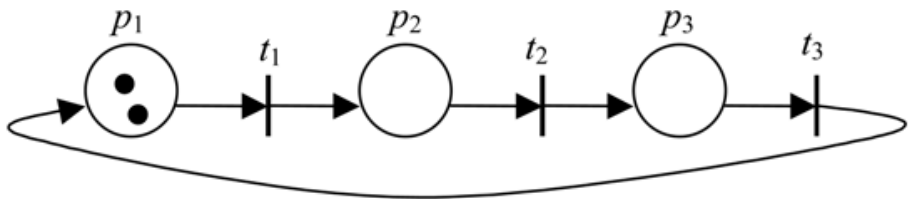

Fig. 1. Petri net example. 
Places, transitions and arcs: Places are represented with circles and transitions are represented with bars. The arcs are directed from places to transitions or from transitions to places. The places contain tokens that travel through the net depending on the firing of a transition. A place $p$ is said to be an input place to a transition $t$ if an arc is directed from $p$ to $t$. Similarly, an output place of $t$ is any place in the net with an incoming arc from transition $t$. In the example (figure 1) $p_{1}$ is an input place of $t_{1}$, and $p_{2}$ is an output place of $t_{1}$.

Transition firing: A transition can fire only if it is enabled. For a transition $t$ to be enabled, all the input places of $t$ must contain at least one token ${ }^{2}$. When a transition is fired, a token is removed from each input place, and one token is added to each output place. In this way the tokens travel through the net depending on the transitions fired.

Definition 2 (Marking) The marking $m_{i}$ of a place $p_{i} \in P$ is a non-negative quantity representing the number of tokens in the place at a given state of the Petri net. The marking of the Petri net is defined as the function $M: P \rightarrow Z_{+}$that maps the set of places to the set of non-negative integers. It is also defined as a vector $M_{j}=\left(m_{1}, m_{2}, \ldots, m|p|\right)$ were $m_{i}=M\left(p_{i}\right)$, which represents the $j_{\text {th }}$ state of the net. $M_{j}$ contains the marking of all the places and the initial marking is denoted by $M_{0}$.

In the example of figure 1 only transition $t_{1}$ is enabled. When $t_{1}$ fires, one token is removed from place $p_{1}$ and one token is added to place $p_{2}$. Figure 2 shows the evolution of the Petri net in the previous example. Figure 2 a) presents the initial marking of the net $M_{0}=$ $\left[M\left(p_{1}\right), M\left(p_{2}\right), M\left(p_{3}\right)\right]=[2,0,0]$, only transition $t_{1}$ is enabled. Figure $2 \mathrm{~b}$ ) presents the net with marking $M_{1}=[1,1,0]$ after $t_{1}$ is fired. Here, transitions $t_{1}$ and $t_{2}$ are enabled and they can be fired. Finally, figure $2 \mathrm{c}$ ) represents the net after $t_{2}$ is fired. In this case transitions $t_{1}$ and $t_{3}$ are enabled with marking $M_{2}=[1,0,1]$.

a)

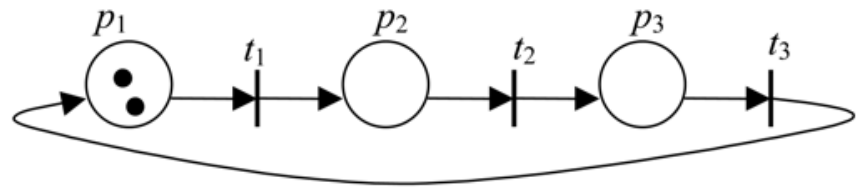

b)

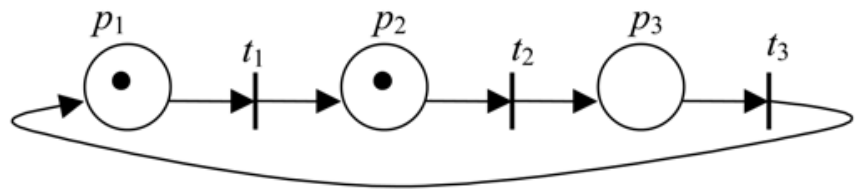

c)

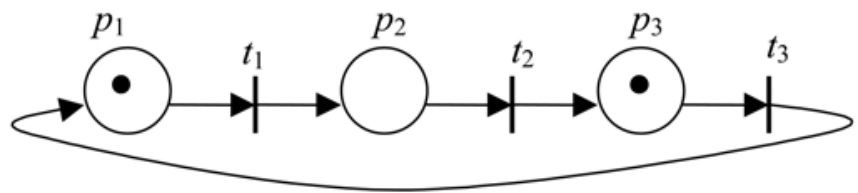

Fig. 2. Petri net evolution after firing transitions $t_{1}$ and $t_{2}$.

2 Assuming the weights $W$ of the Petri net are equal to one. When the weights are not indicated they are assumed to be one. The weight on an arc coming to a transition from one of the incoming places indicates the minimum number of tokens needed in the incoming place in order for that transition to be enabled. When the transition fires, it will remove from the incoming place the amount of tokens indicated by the weight of the arc. 
The marking of the Petri net represents the state of the net. As described above, the transitions change the state of the Petri net in the same way an event changes the state of a DEDS.

Definition 3 (Reachability graph) The reachability graph has the marking of the Petri net (or state of the Petri net) as a node. An arc of the graph joining $M_{i}$ with $M_{j}$ represents the transition when firing takes the Petri net from the marking (state) $M_{i}$ to the marking $M_{j}$.

The reachability graph of the Petri net in figure 1 is presented in figure 3.

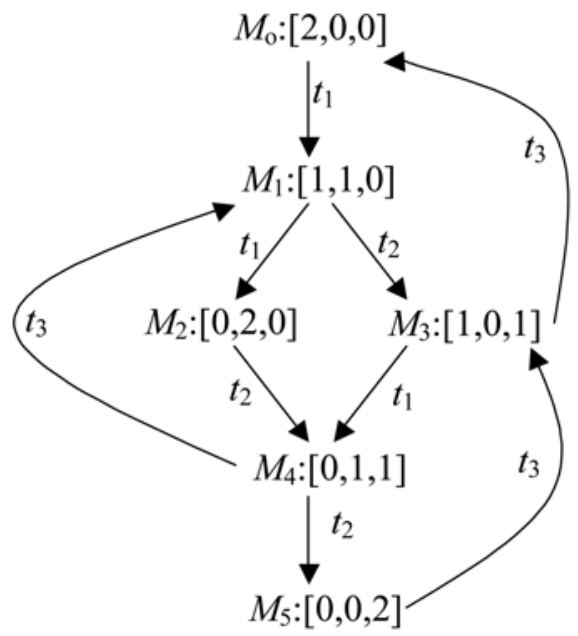

Fig. 3. Reachability graph.

\subsection{Properties}

This section covers some of the most important properties of Petri nets such as Reachability, Liveness, Boundedness and Reversibility. These properties are essential for the analysis of Petri net models. Furthermore, they are required characteristics for the use of Petri nets in performance evaluation [8][10][11].

These are properties that could be applied to multi-agent systems models. Examples of these properties are boundedness and liveness since they are related to deadlock avoidance in DEDS. Other properties are going to be relevant to multi-agent systems particularly to the communication, interaction, and single agent architectures. It is unknown how the available properties of Petri nets relate to models of multi-agent systems. This is a research question addressed in this study. In addition, the definition of new properties might be required to capture behaviors particular to multi-agent systems. A complete description of the available Petri net properties can be found in [8]. The analysis methods developed in this research will focus on the following properties.

Definition 4 (Reachability) A marking $M_{j}$ is said to be reachable from marking $M_{i}$ if there exists a sequence of transitions that takes the Petri net from state $M_{i}$ to $M_{j}$. The set of all possible markings that are reachable from $M_{0}$ is called the reachability set and is defined by $R\left(M_{0}\right)$.

The concept of reachability is essential for the study of the dynamic properties of a Petri net. The reachability set can be obtained from the reachability graph presented previously, e.g., figure $3[8][11]$. 
Definition 5 (Liveness) A Petri net is said to be live for a marking $M_{0}$ if for any marking in $R\left(M_{0}\right)$ it is possible to fire a transition.

The liveness property guaranties the absence of deadlock in a Petri net. This property can also be observed from the reachability graph. If the reachability graph contains an absorbent state $^{3}$, then the Petri net is not live at that state and it is said to have a deadlock [8][11].

Definition 6 (Boundedness) A Petri net is said to be bounded or k-bounded if the number of tokens in each place does not exceed a finite number $k$ for any marking in $R\left(M_{0}\right)$. Furthermore, a Petri net is structurally bounded if it is bounded for any finite initial marking $M_{0}$. A Petri net is said to be safe if it is 1-bounded [8].

Definition 7 (Reversibility) A Petri net is reversible, if for any marking in $R\left(M_{0}\right), M_{0}$ is reachable. This means that the Petri net can always return to the initial marking $M_{0}$ [8][11].

For the example in figure 1 we have a reachability set $R\left(M_{0}\right)=\left\{M_{1}=[1,1,0], M_{2}=[0,2,0], M_{3}\right.$ $\left.=[1,0,1], M_{4}=[0,1,1], M_{5}=[0,0,2]\right\}$. The Petri net is live, reversible and 2-bounded for the marking $M_{0}=[2,0,0]$.

\subsection{Structural analysis}

This section considers the structural analysis of Petri nets by using invariant analysis as described in [8][13]. Basically, the liveness and boundedness of the net will be assessed by using P-invariants and T-invariants. These invariants are obtained from the incidence matrix of the net and they give information regarding token conservation and transition firing sequences that leave the marking of the net unchanged. These concepts are used to assess the overall liveness and boundedness of the net.

Definition 8 (Incidence matrix) Let $a_{i j}^{+}=w(i, j)$ be the weight of the arc that goes from transition $t_{i}$ to place $p_{j}$ and $a_{i j}^{-}=w(j, i)$ be the weight of the arc from place $p_{j}$ to transition $t_{i}$. The incidence matrix A of a Petri net has $|T|$ number of rows and $|P|$ number of columns. It is defined as $A=\left[a_{i j}\right]$ where $a_{i j}=a_{i j}^{+}-a_{i j}^{-}$.

The example presented in figure 1 shows an ordinary Petri net (all the weights are equal to 1) and the following is its corresponding incidence matrix.

$$
A_{1}=\left[\begin{array}{rrr}
-1 & 1 & 0 \\
0 & -1 & 1 \\
1 & 0 & -1
\end{array}\right]
$$

Definition 9 (Net-invariants) Let A be the incidence matrix. A P-invariant is a vector that satisfies the equation

$$
A x=0
$$

and a T-invariant is a vector that satisfies the equation

$$
A^{T} y=0 .
$$

\footnotetext{
${ }^{3}$ If the net is not live for marking $M_{0}$ then at least one marking from $R\left(M_{0}\right)$ will not have any enabled outgoing transitions. If the reachability graph is considered as the state graph of the net, then an absorbent state is that from which the marking it is representing does not have any outgoing transitions enabled. As a result, when the net reaches an absorbent state, it will remain in it indefinitely.
} 


\subsubsection{Boundedness assessment}

The P-invariants of the incidence matrix are used in Theorem 1 to make an assessment of the boundedness of the Petri net. A Petri net model is covered by P-invariants if and only if, for each place $s$ in the net, there exists a positive P-invariant $x$ such that $x(s)>0$.

Theorem 1 A Petri net is structurally bounded if it is covered by P-invariants and the initial marking $M_{0}$ is finite [13].

\subsubsection{Liveness assessment}

The liveness of the Petri net model is assessed on Theorem 2 by means of the T-invariants of the incidence matrix. A Petri net model is covered by T-invariants if and only if, for each transition $t$ in the net, there exists a positive T-invariant $y$ such that $y(t)>0$. This is a necessary condition but not sufficient. The liveness assessment by the use of T-invariants is still an open problem [8].

Theorem 2 A Petri net that is finite is live and bounded if it is covered by T-invariants [13].

\section{Modeling of multi-agent systems with indirect interaction}

The methodology presented here consists of defining a simple multi-agent system based on the abstract architecture for intelligent agents $(M)$. The abstract architecture is modeled as a discrete-event system using Petri nets $(N)$ and structural and reachability analysis provides an assessment of the interaction properties. Deadlock avoidance in the multiagent system is considered as a key property, and it is evaluated using the liveness and boundedness properties of the Petri net model.

The purpose of this work consists of the definition of an abstract architecture for multiagent systems with indirect interaction, analogous to the abstract architecture for intelligent agents. The proposed architecture allows the description of agent-to-agent interactions via changes in the environment and serves as an initial description of the discrete-event dynamics of the multi-agent system. In addition, this work presents an algorithm (algorithms 1 and 2) to obtain a Petri net model of a multi-agent system by making use of the multi-agent system's abstract architecture. Finally, a methodology to ensure that the multi-agent system is deadlock free is presented; it is based on the analysis of the properties of the Petri net model.

Here, the abstract architecture of intelligent agents is used as a starting point; particularly the abstract architecture for purely reactive agents. The level of abstraction of agents modeled by the abstract architecture makes it a good candidate for the study of multiagent systems as discrete-event dynamic systems. The study of interaction frameworks is first approached by studying the simplest means of interaction among agents (indirect interaction); assuming the agents have the perception/action capabilities and agents can interact by changing each others environment.

Modeling approach based on interaction among agents: The interactions between agents can be either a direct agent-to-agent interaction or an indirect interaction. The typical structure of a multi-agent system was presented in [14] and is reproduced in figure 4 . It shows how the agents interact among each other and how they operate over a metalevel (multi-agent level) environment. Arrows define direct agent interactions from agent to agent; the indirect interactions are based on the environment. In the indirect interaction, an agent modifies another agent's environment triggering a reaction. The indirect interaction occurs in the cases when two or more agents share a subset of the environment. It should be 
noted that the overall multi-agent system acts over a meta-level environment. An agent that is part of the multi-agent system has its own environment that is somehow related to the meta-level environment of the multi-agent system. This meta-level environment of the multi-agent system is referred to in the literature as being an open environment [15]. A complex problem will provide an open environment, which is dynamic, has components that are unknown in advance, its structure changes over time and might be heterogeneous in its implementation [15]. By focusing on the interactions among agents as described above, it is natural to regard a multi-agent system as a discrete-event system.

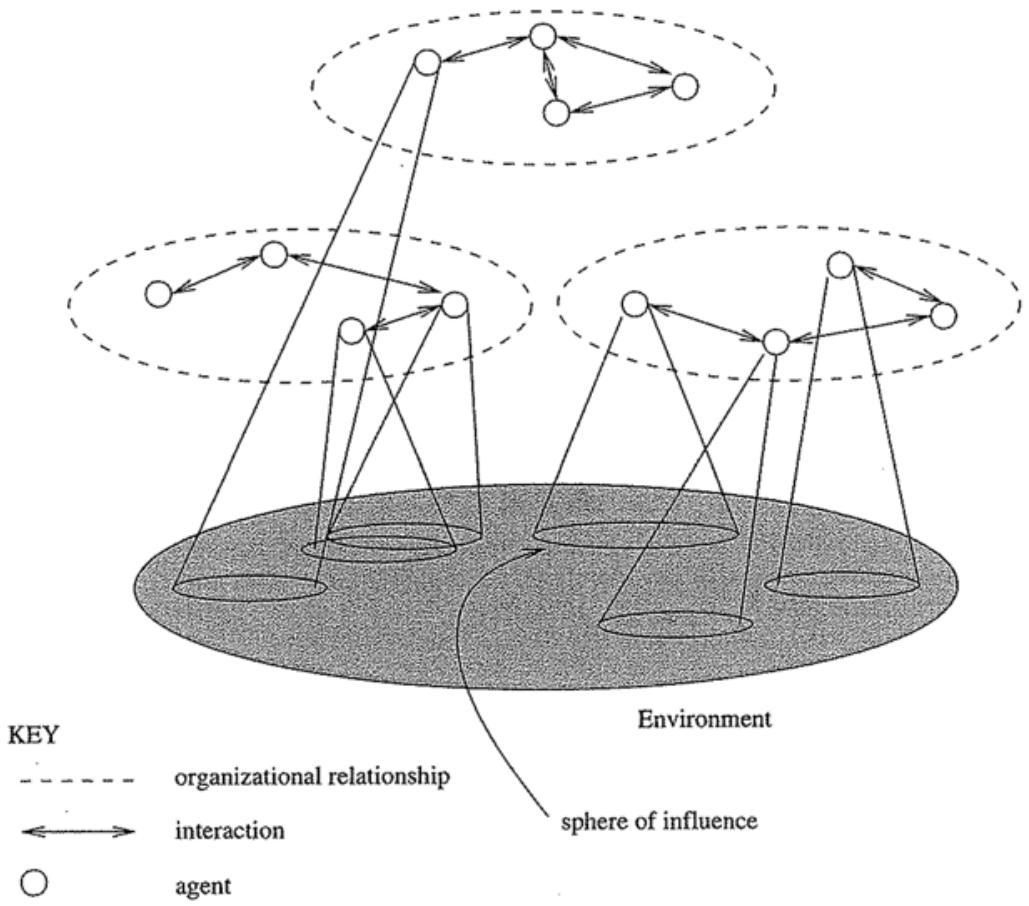

Fig. 4. Structure of a multi-agent system [14].

Following the goal of defining a methodology for modeling multi-agent systems, there is a need to define a modeling methodology that makes no use of the context of the system or at least abstracts itself from it. Basically, looking at an abstract architecture as a means of modeling single agents and multi-agent systems. The proposed modeling approach, uses the abstract architecture to obtain the Petri net models.

\subsection{Petri net models from the abstract architecture}

The artificial intelligence research considers three difierent paradigms for intelligent agents: a) reactive and b) deliberative; and c) hybrids between them. The abstract architecture models how an agent behaves with respect to changes in its environment. Here, an agent has its own environment and this environment is defined by the nature of the agent. The goals, objectives and the general purpose of the agent define its environment. This abstract architecture is based on the reactive paradigm of perception and action. A purely reactive 
agent has a perception of the environment and it is used in the decision mechanism that provides an action in the agent. A reactive agent can also have an internal state as a decision mechanism for the actions to be undertaken. An agent with perception and internal state capabilities has more computational power than an agent without them and its computational power is now comparable with that of the Belief-Desired-Intention architecture as described in [14].

Abstract model for purely reactive agents: In a purely reactive agent, the perception part records the changes in the state of the environment. The action part computes the actions to be taken in order to react to changes in the environment. The agent environment changes based on the actions applied by the agent, as well as actions by other agents, and it may be dynamic in that it may change by itself.

The environment consists of a set of states $S=\left\{s_{1}, s_{2}, \ldots\right\}$. The agent can undertake a set of actions $A=\left\{a_{1}, a_{2}, \ldots\right\}$ and perceive a set of percepts $P=\left\{p_{1}, p_{2}, \ldots\right\}$. For a purely reactive agent, the behavior of the agent can be represented as the function action: $P \rightarrow A$ and perception : $S \rightarrow P$. The deterministic behavior of an environment can be represented by the function environment : $S \times A \rightarrow S$.

Petri net modeling of multi-agent systems: A Petri net is defined as a five-tuple $(P, T$, $\left.A, W, M_{0}\right)$ where: $P$ is a finite set of places, $T$ is a finite set of transitions, $A \subseteq(P \times T)[(T \times P)$ is a set of $\operatorname{arcs}, W: A \rightarrow\{1,2,3, \ldots]$ is a weight function, and $M_{0}: P \rightarrow Z_{+}^{|P|}$ is the initial marking. When modeling, several interpretations can be assigned to places and transitions. For a discrete-event dynamic system a transition is regarded as an event, and the places are interpreted as a condition for an event to occur.

There are properties we would like to show for multi-agent systems. Examples of these properties are boundedness and liveness since they are related to deadlock avoidance in discrete-event systems. Other properties are going to be relevant to multi-agent systems particularly to the communication, interaction and individual agent architectures. The reachability, liveness and boundedness properties of Petri nets are going to be used in the analysis presented in this chapter.

\subsubsection{Obtaining Petri net models from the abstract architecture}

Places model the environmental state of the agent. Having a token in a place representing state $s_{i}$ means that the agent is currently in such a state.

Transitions model the actions of an agent. The environmental state is changed by actions so, for the Petri net model having tokens move from one place to another by firing transitions, this agrees with the execution process of the abstract architecture.

Algorithm 1 (Petri net submodel for agent $i$ ) Let $S_{i}$ be the set of environmental states of agent $i$, and $s_{i j} \in S_{i}$ be the $j^{\text {th }}$ environmental state of agent $i$. Similarly, let $A_{i}$ be the set of actions of agent $i$, and $a_{i k} \in A_{i}$ be the $k^{\text {th }}$ action of agent $i$.

1. Add a place for each element of the environment $S_{i}$ and label each place using notation $P_{i j}$ for $s_{i j}$.

2. Add a transition for each action in $A_{i}$ and label each transition using notation $T_{i k}$ for $a_{i k}$.

3. For each instance of the function environment: $S_{i} \times A_{i} \rightarrow S_{i}$ say $s_{i j} \times a_{i k} \rightarrow s_{i l}:$ a) add an arc leaving from place $P_{i j}$ and ending in transition $T_{i k} ; b$ ) add an arc leaving from transition $T_{i k}$ and ending in place $\left.P_{i l} ; c\right)$ add a weight of 1 to each arc.

If an arc from transition $T_{i k}$ to place $P_{i l}$ already exists, add a new transition and label it $T^{\prime}{ }_{i k}$; perform this step using $T^{\prime}{ }_{i k}$ instead of $T_{i k}$.

4. Add a token in the place representing the initial state of the environment. 
Algorithm 2 (Petri net model of the multi-agent system) The Petri net sub-models of each of the individual agents in the system should be joined based on their indirect interactions. In general, this indirect interaction will be in such a way that an agent $i$ action will change an environment state of agent $j$. This communication act can be regarded as a regular action in the construction of the complete model. There will be arcs added from the places modeling the environmental states of agent $j$ to the transition modeling the communication in agent $i$.

\subsubsection{Analysis of the Petri net model}

The Petri net model of the multi-agent system can be analyzed to assess system properties like deadlock. Inspection of the reachability graph of the Petri net model can indicate if the model is live and bounded. On the other hand, liveness and boundedness properties can also be assessed using invariant analysis [13]. Basically, the liveness and boundedness of the net can be assessed by using the P-invariants and T-invariants obtained from the incidence matrix which give information regarding token conservation and transition firing sequences that leave the marking of the net unchanged.

Let $a_{i j}^{+}=w(i, j)$ be the weight of the arc that goes from transition $t_{i}$ to place $p_{j}$ and $a_{i j}^{-}=w(j, i)$ be the weight of the arc from place $p_{j}$ to transition $t_{i}$. The incidence matrix $A$ of a Petri net has $|T|$ number of rows and $|P|$ number of columns. It is defined as $A=\left[a_{i j}\right]$ where $a_{i j}=a_{i j}^{+}-a_{i j}^{-}$. Furthermore, a P-invariant is a vector that satisfies $A x=0$ and a T-invariant is a vector that satisfies $A^{T} y=0$.

A Petri Net model is covered by P-invariants if and only if for each place $s$ in the net, there exists a positive P-invariant $x$ such that $x(s)>0$. Furthermore, a Petri net is structurally bounded if it is covered by P-invariants and the initial marking $M_{0}$ is finite. In addition, a Petri net model is covered by T-invariants if and only if for each transition $t$ in the net, there exists a positive T-invariant $y$ such that $y(t)>0$. Furthermore, a Petri Net is live and bounded if it is covered by T-invariants. This is a necessary condition but not sufficient.

\subsection{Multi-agent system modeling and analysis example}

This section presents a description of a simple multi-agent system consisting of two physical agents that work together at a task. This system will be used throughout this chapter to illustrate how the Petri net and abstract architecture models can be applied to multi-agent systems.

This system consists of two agents referred to as agent $A$ and agent $B$. They move objects from one end of a path to the other. Figure 5 shows 4 scenarios in the operation of the system. Both agents are capable of moving objects and agent A moves faster than agent $\mathrm{B}$. The objective of agent $A$ is to go to the end of the path, pick an object, and return to the start of the path (figure 5a). Since it is faster than agent B it reaches the objects first (figure $5 b$ ). At the time they intersect in the path, agent $A$ gives its object to agent $B$ and returns to the end of the path to pick another object (figures $5 \mathrm{c}$ and $5 \mathrm{~d}$ ). On the other hand, the objective of agent $B$ is to go in the direction of the end of the path, intersect with agent $A$ and get its object. Once the agent has an object, it returns to the start of the path, places the object down and starts all over again.

The meta-level environment of the system consists of a single path with a set of objects on one side and no objects on the other side.

There is indirect interaction between the agents via changes in their environments. The exchange of the object between the agents should be regarded as a result of a change in the 
environment of both agents. The two agents are regarded as purely reactive, which implies they do not have a record of history and they do not have an internal state. The decisions they make are about which actions to undertake and those decisions are directly influenced by the location of the agent in the path and whether the agent has an object or not. It should be noted also, that the goal of the overall multi-agent system is a little different from the goal of each specific agent.

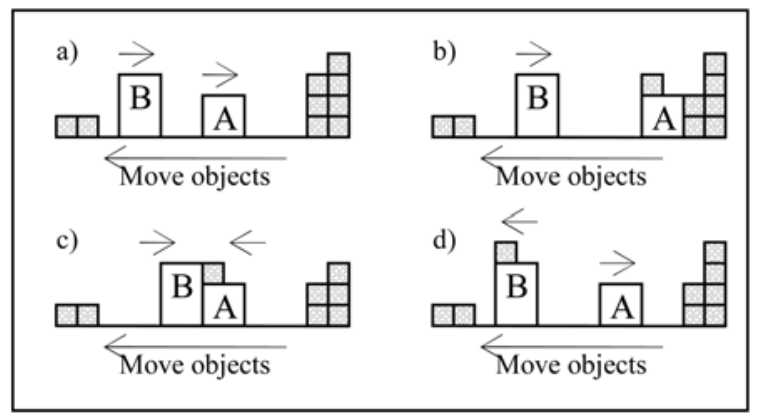

Fig. 5. Multi-agent system example for modeling and analysis.

\subsubsection{Abstract architecture description}

Under normal conditions, agent A will be walking to the end of the path to pick the object to be moved. The object is going to be taken away by the other agent at the intersection. Let $M$ be the multi-agent system with agent $\mathrm{A}$ and $\mathrm{B}$ modeled with the abstract architecture. Agent A has environmental states $S_{A}$, a set of actions $A_{A}$, an action function action $_{A}$, and an environment evolution function environment $A_{A}$. On the other hand, Agent $B$ has environmental states $S_{B}$, a set of actions $A_{B}$, an action function action ${ }_{B}$, and an environment evolution function environment $t_{B}$. Tables 2 and 3 describe the possible environmental states $\left(S_{A}\right)$ and the actions $\left(A_{A}\right)$ that agent $\mathrm{A}$ can undertake.

\begin{tabular}{|c|l|}
\hline State $\left(S_{A}\right)$ & Description \\
\hline$s_{1}$ & Agent has object \\
$s_{2}$ & Agent has no object and it is not at the end \\
$s_{3}$ & Agent has no object and it is at the end \\
\hline
\end{tabular}

Table 2. Environmental states of agent A of $M$.

\begin{tabular}{|c|l|}
\hline Actions $\left(A_{A}\right)$ & Description \\
\hline$a_{1}$ & Walk to the end \\
$a_{2}$ & Pick an object \\
$a_{3}$ & Walk to start \\
\hline
\end{tabular}

Table 3. Actions for agent A of $M$.

Table 4 presents the mapping of the environment (environment $t_{A}$ ) describing how it will be changing as the agent undertakes actions. It should be noted that the notion of exchanging the part with agent $B$ at the intersection has not been considered explicitly in the description of the environment $t_{A}: S \times A \rightarrow S$ for agent $\mathrm{A}$. The agents decision mechanism is described by the action $_{A}$ function as presented in (4). 


$$
\operatorname{action}_{A}(s)=\left\{\begin{array}{ll}
a_{1} & \text { if } s=s_{2} \\
a_{2} & \text { if } s=s_{3} \\
a_{3} & \text { if } s=s_{1}
\end{array}\right\}
$$

Agent $B$ will be walking toward the end of the path until it intersects with agent $A$ which is on its way back to the beginning of the path carrying an object. At the intersection point, agent $B$ takes over the object of agent $A$ and proceeds to return to the beginning of the path to drop the object and start the cycle again. The abstract architecture of agent $B$ is presented in Tables 4 and 5, and the $\operatorname{action}_{B}(s)$ function is described in (5).

\begin{tabular}{|c|c|c|c|}
\hline$A_{A}$ & $S_{A}$ & $S_{A}$ & Description \\
\hline$a_{1}$ & $s_{2}$ & $s_{3}$ & $\begin{array}{l}\text { Walking towards the end of the path. Eventually will } \\
\text { reach the end with no object. }\end{array}$ \\
\hline$a_{2}$ & $s_{3}$ & $s_{1}$ & Now the agent has an object. \\
\hline$a_{3}$ & $s_{1}$ & $s_{1}$ & $\begin{array}{l}\text { Walking to the start of the path. Eventually will lose } \\
\text { object to agent B. }\end{array}$ \\
\hline
\end{tabular}

Table 4. Environment function for agent A of $M$.

\begin{tabular}{|c|l|}
\hline State $\left(S_{B}\right)$ & Description \\
\hline$s_{1}$ & Agent has no object \\
$s_{2}$ & Agent has object and it is not at the start \\
$s_{3}$ & Agent has object and it is at the start \\
$s_{4}$ & Agent is at the intersection with agent A \\
\hline
\end{tabular}

Table 5. Environmental states for agent B of $M$.

\begin{tabular}{|c|l|}
\hline Actions $\left(A_{B}\right)$ & Description \\
\hline$a_{1}$ & Walk to the end \\
$a_{2}$ & Take over object from agent A \\
$a_{3}$ & Walk to start \\
$a_{4}$ & Put object down at start \\
\hline
\end{tabular}

Table 6. Actions for agent B of $M$.

$$
\operatorname{action}_{B}(s)=\left\{\begin{array}{ll}
a_{1} & \text { if } s=s_{1} \\
a_{2} & \text { if } s=s_{4} \\
a_{3} & \text { if } s=s_{2} \\
a_{3} & \text { if } s=s_{3}
\end{array}\right\}
$$

The mapping of the environment of agent B (environment ${ }_{B}$ ) is presented in Table 7. The interaction with agent $\mathrm{A}$ (in the exchange of the part) is implicitly modeled by action $a_{2}$ although there is no indication in its abstract architecture that it will change the environment of agent $\mathrm{A}$.

\subsubsection{Petri net model}

The Petri net model of the multi-agent system was obtained following the procedure described in algorithm 1 (Petri net submodel for an agent) and algorithm 2 (Petri net model of the multi-agent system). Basically, algorithm 1 is executed once for each agent in the system. 


\begin{tabular}{|c|c|c|c|}
\hline$A_{B}$ & $S_{B}$ & $S_{B}$ & Description \\
\hline$a_{1}$ & $s_{1}$ & $s_{4}$ & $\begin{array}{l}\text { Will walk toward the end until intersection } \\
\text { with agent } A\end{array}$ \\
\hline$a_{2}$ & $s_{4}$ & $s_{2}$ & Object exchange, now it has an object \\
\hline$a_{3}$ & $s_{2}$ & $s_{3}$ & Will walk towards start until it reaches it \\
\hline$a_{4}$ & $s_{3}$ & $s_{1}$ & Will drop the object at start \\
\hline
\end{tabular}

Table 7. Environment function for agent $\mathrm{B}$ of $M$.

Furthermore, once all the individual agents' submodels are obtained, algorithm 2 is used to join the submodels of those agents that engage in indirect interaction.

Petri net submodel for agent A: The Petri net submodel for agent A presented in figure 6 is obtained as follows. Step 1 of the algorithm is concerned with the places of the Petri net. For each of the environmental states of agent $\mathrm{A}$ as described in table 2, a place is added to the new submodel following the described notation. In this step, three places are added in total, e.g., place $p_{A 2}$ which models the environmental state $s_{2}$. In this state, agent A does not have an object and it is not at the end of the path. The transitions of the model are added in the second step of the algorithm. For this agent, a total of three transitions are added which model the three actions agent A can undertake, e.g., transition $t_{A 2}$ models action $a_{2}$ which in turn is the pick an object action of agent A as described in table 3. The arcs of the Petri net are added in Step 3 of the algorithm. These arcs are related directly to the function that describes the evolution of the environment of the agent. This function maps the Cartesian product of environmental states and actions into the environmental states resulting from the agent undertaking a particular action. From the Petri net model point of view, this means a Cartesian product of places and transitions that is mapped into a set of places. The addition of arcs revolves around the transitions/actions of each instance of this mapping as described in table 4, e.g., $f\left(s_{2}, a_{1}\right)=s_{3}$ indicates that two arcs should be added to transition $t_{A 1}$, an incoming arc from place $p_{A 2}$ and an outgoing arc to place $p_{A 3}$. The last step of the algorithm consists of assigning the initial condition or current state to the model. A token is added to the place representing the current environmental state of the agent. A complete list with description of places and transitions is presented in table 8 .

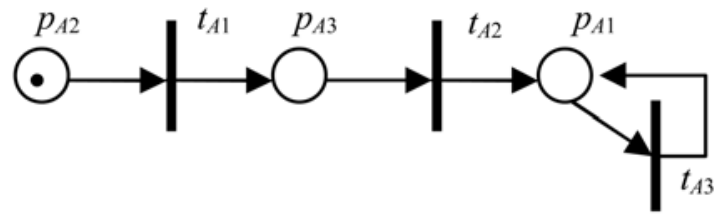

Fig. 6. Petri net model of agent A.

The Petri net submodel for agent $\mathrm{A}$ is not pure since place $p_{A 1}$ and transition $t_{A 3}$ form a selfloop. A Petri net is said to be pure if there is no place $p$ that is both the input and output place to a transition $t$ [8]. This self-loop is an artifact of the abstract architecture model of the agent. A token in place $p_{A 1}$ indicates that the agent is currently at environmental state $S_{1}$, which in turn means that agent $A$ has an object. Furthermore, transition $t_{A 3}$ models action $a_{3}$ which indicates that agent $A$ walks to the start of the path. This self-loop models agent A walking towards the start of the path while carrying an object. It does not model what will happen once the agent reaches the start of the path. It must be noted that this is not a deficiency in the Petri net construction model, but a choice made in generating the abstract 
architecture description for this agent. This description assumes that when agent $\mathrm{A}$ is holding an object and walking towards the start of the path, the other agent will intersect with it and take over the object.

From the Petri net analysis point of view it can be seen that a token will eventually reach place $p_{A 1}$ and remain there indefinitely. This is consistent with the abstract architecture description since it does not model the capabilities of the agent once it reaches the start of the path having an object to drop there. As a result, the dynamics of this agent by itself reach a stationary state. A stationary state in this context means that the agent will keep doing the same activity and that the Petri net model indicates that the dynamics eventually get trapped at one state. It can be seeen from the reachability graph in figure 7 that the agent reaches state $M_{2}$ and remains there. The only transition enabled at state $M_{2}$ is transition $t_{A 3}$ and once it fires, the system remains in state $M_{2}$.

The states of the reachability graph are described in figure 7 as well. The initial marking $M_{0}$ describes the initial condition where agent $\mathrm{A}$ has no object and it is not at the end of the path. From the reachability graph it can be concluded that the net is bounded and live for $M_{0}=[0,1,0]$. Even though the subnet is live, it will remain in state $M_{2}$ once such a state is reached, as a result, the subnet is not reversible.

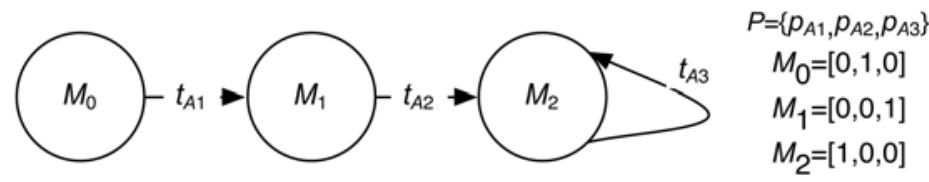

Fig. 7. Reachability graph of agent A.

The incidence matrix $A_{A}$ of the Petri net submodel of agent $\mathrm{A}$ is presented in equation 6 . The order of the places in the matrix is $P=\left\{p_{A 1}, p_{A 2}, p_{A 3}\right\}$ and the order for transitions is $T=\left\{t_{A 1}, t_{A 2}, t_{A 3}\right\}$. The incidence matrix $A_{A}$ is a $m \times n$ matrix, where $m=|T|$ and $n=|P|$. Let $x$ be a P-invariant of the subnet which satisfies equation $A x=0, x_{1}=[1,1,1]^{T}$ is the only Pinvariant of $A_{A}$ with integer elements. Furthermore, let $y$ be a T-invariant of the subnet which satisfies equation $A^{T} y=0, y_{1}=[0,0,1]^{T}$ is the only T-invariant of $A_{A}$ with integer elements.

$$
A_{A}=\left[\begin{array}{rrr}
0 & 1 & 0 \\
-1 & 0 & 0 \\
1 & -1 & 0
\end{array}\right]
$$

The Petri net submodel of agent A is not covered by positive T-invariants. As a result, the necessary condition for liveness is not met and it can be concluded that the subnet is not structurally live. On the other hand, the subnet is covered by positive P-invariants, as a result, the subnet is bounded for finite initial markings.

Petri net submodel of agent B: The Petri net submodel of agent B is presented in figure 8 . This submodel is obtained in a similar way as that of agent A, following the steps presented in algorithm 1 and the abstract architecture description of the agent presented in the previous section. The first step of the algorithm results in the addition of four places to the model based on the set of environmental states described in table 5 . The four transitions are added in step 2, representing the set of actions in table 6. The four instances of the environment evolution function presented in table 7 result in the eight arcs of the model. The token was added to place $p_{B 1}$ to indicate that the agent does not have an object. 


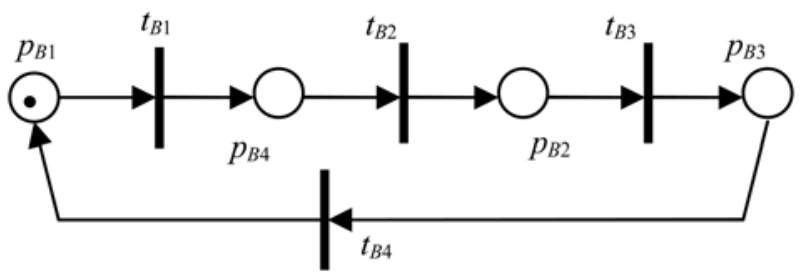

Fig. 8. Petri net model of agent B.

The reachability graph is presented in figure 9. The initial marking $M_{0}$ describes the initial condition where agent $B$ has no object. From the reachability graph it can be concluded that the net is bounded and live for $M_{0}=[1,0,0,0]$.

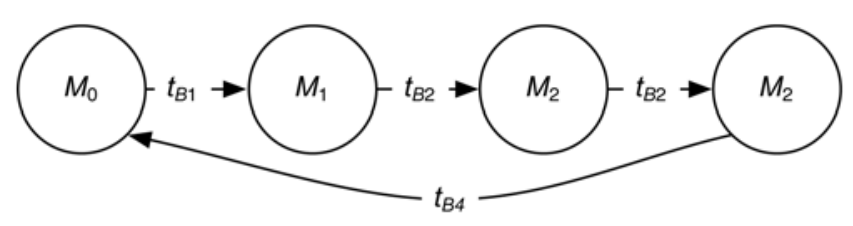

$$
\begin{aligned}
P=\left\{p_{B 1}, p_{B 2}, p_{B 3}, p_{B 4}\right\} & \\
M_{0} & =[1,0,0,0] \\
M_{1} & =[0,0,0,1] \\
M_{2} & =[0,1,0,0] \\
M_{3} & =[0,0,1,0]
\end{aligned}
$$

Fig. 9. Reachability graph of agent $B$.

The incidence matrix $A_{B}$ of the Petri net submodel of agent $\mathrm{B}$ is presented in equation 7 . The order of the places in the matrix is $P=\left\{p_{B 1}, p_{B 2}, p_{B 3}, p_{B 4}\right\}$ and the order for transitions is $T=$ $\left\{t_{B 1}, t_{B 2}, t_{B 3}, t_{B 4}\right\}$. The incidence matrix $A_{B}$ is a $m \times n$ matrix, where $m=|T|$ and $n=|P|$. Let $x$ be a P-invariant of the subnet which satisfies equation $A x=0, x_{1}=[1,1,1,1]^{T}$ is the only Pinvariant of $A_{B}$ with integer elements. Furthermore, let $y$ be a T-invariant of the subnet which satisfies equation $A^{T} y=0, y_{1}=[1,1,1,1]^{T}$ is the only T-invariant of $A_{B}$ with integer elements.

The Petri net submodel of agent B is covered by positive T-invariants. As a result, the necessary condition for liveness is met so it can be structurally live. On the other hand, the subnet is also covered by positive P-invariants, as a result, the subnet is bounded for finite initial markings.

$$
A_{B}=\left[\begin{array}{rrrr}
-1 & 0 & 0 & 1 \\
0 & 1 & 0 & -1 \\
0 & -1 & 1 & 0 \\
1 & 0 & -1 & 0
\end{array}\right]
$$

Petri net model of the complete multi-agent system: Let $N=\left(P, T, A, W, M_{0}\right)$ be the Petri net model of the complete system with places $P=\left\{p_{A 1}, p_{A 2}, p_{A 3}, p_{B 1}, p_{B 2}, p_{B 3}, p_{B 4}\right\}$, transitions $T=$ $\left\{t_{A 1}, t_{A 2}, t_{A 3}, t_{B 1}, t_{B 2}, t_{B 3}, t_{B 4}\right\}$, and $M_{0}=[0,1,0,1,0,0,0]$. Figure 10 shows $N$ and the interpretation of places and transitions is presented in Table 8 .

This model was obtained by joining the Petri net submodels of the two agents, by following the methodology presented in algorithm 2. The premise of the algorithm is to identify agents engaging in indirect interaction in order to join their models. Furthermore, a transition firing from one of the agents will modify the other agent's environmental state. For this example, transition $t_{B 2}$ which models an action of agent $B$, modifies the environmental state of agent A. The interpretation in terms of the system's description is 
that when agent B takes over the object from agent $A$, the environmental state of agent $\mathrm{A}$ changes from having an object to not having an object and not being at the end of the path. As a result, two additional arcs are added to the submodels in order to construct the overall multi-agent system model; an incoming arc to transition $t_{B 2}$ from place $P_{A 1}$, and an outgoing arc from transition $t_{B 2}$ to place $p_{A 2}$.

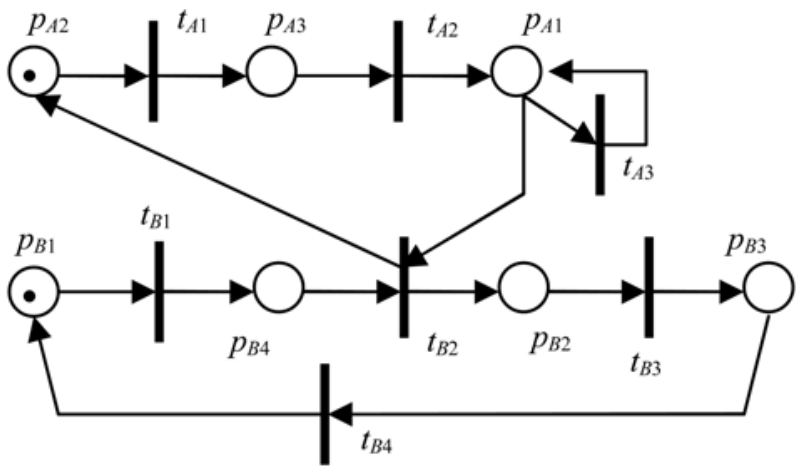

Fig. 10. Petri net model $N$.

\begin{tabular}{|c|c|c|}
\hline Element & Name & Description \\
\hline Places $(P)$ & $\begin{array}{l}p_{A 1} \\
p_{A 2} \\
p_{A 3} \\
p_{B 1} \\
p_{B 2} \\
p_{B 3} \\
p_{B 4}\end{array}$ & $\begin{array}{l}\text { A token in this place indicates the environment is at } \\
\text { state } s_{1} \text { for agent A } \\
\text { A token in this place indicates the environment is at } \\
\text { state } s_{2} \text { for agent A } \\
\text { A token in this place indicates the environment is at } \\
\text { state } s_{3} \text { for agent A } \\
\text { A token in this place indicates the environment is at } \\
\text { state } s_{1} \text { for agent B } \\
\text { A token in this place indicates the environment is at } \\
\text { state } s_{2} \text { for agent B } \\
\text { A token in this place indicates the environment is at } \\
\text { state } s_{3} \text { for agent B } \\
\text { A token in this place indicates the environment is at } \\
\text { state } s_{4} \text { for agent B }\end{array}$ \\
\hline Transitions $(T)$ & $\begin{array}{l}t_{A 1} \\
t_{A 2} \\
t_{A 3} \\
t_{B 1} \\
t_{B 2} \\
t_{B 3} \\
t_{B 4}\end{array}$ & $\begin{array}{l}\text { Execution of action } a_{1} \text { for agent A } \\
\text { Execution of action } a_{2} \text { for agent A } \\
\text { Execution of action } a_{3} \text { for agent A } \\
\text { Execution of action } a_{1} \text { for agent B } \\
\text { Execution of action } a_{2} \text { for agent B } \\
\text { Execution of action } a_{3} \text { for agent B } \\
\text { Execution of action } a_{4} \text { for agent B }\end{array}$ \\
\hline
\end{tabular}

Table 8. Description of Petri net model $N$.

The tokens in places $p_{A 2}$ and $p_{B 1}$ represent the initial conditions of agent $\mathrm{A}$ and $\mathrm{B}$ respectively. The tokens travel through the net representing different environment states for the agents as the system executes. The systems execution scenarios presented in figure 5 can 
be identified in the Petri net model, e.g., scenario d) of figure 5 will be represented in the Petri net model as having a token in $p_{A 2}$ and $p_{B 2}$. The Petri net model can now be analyzed to assess the deadlock property in a systematic way.

\subsubsection{Analysis of the Petri net model $\mathbf{N}$}

The Petri net model presented in figure $10(N)$ can now be analyzed to assess the deadlock property of the underlying multi-agent system. The reachability graph of the model is presented in figure 11 and it shows that the net is live and bounded. Therefore, the multiagent system is deadlock free.

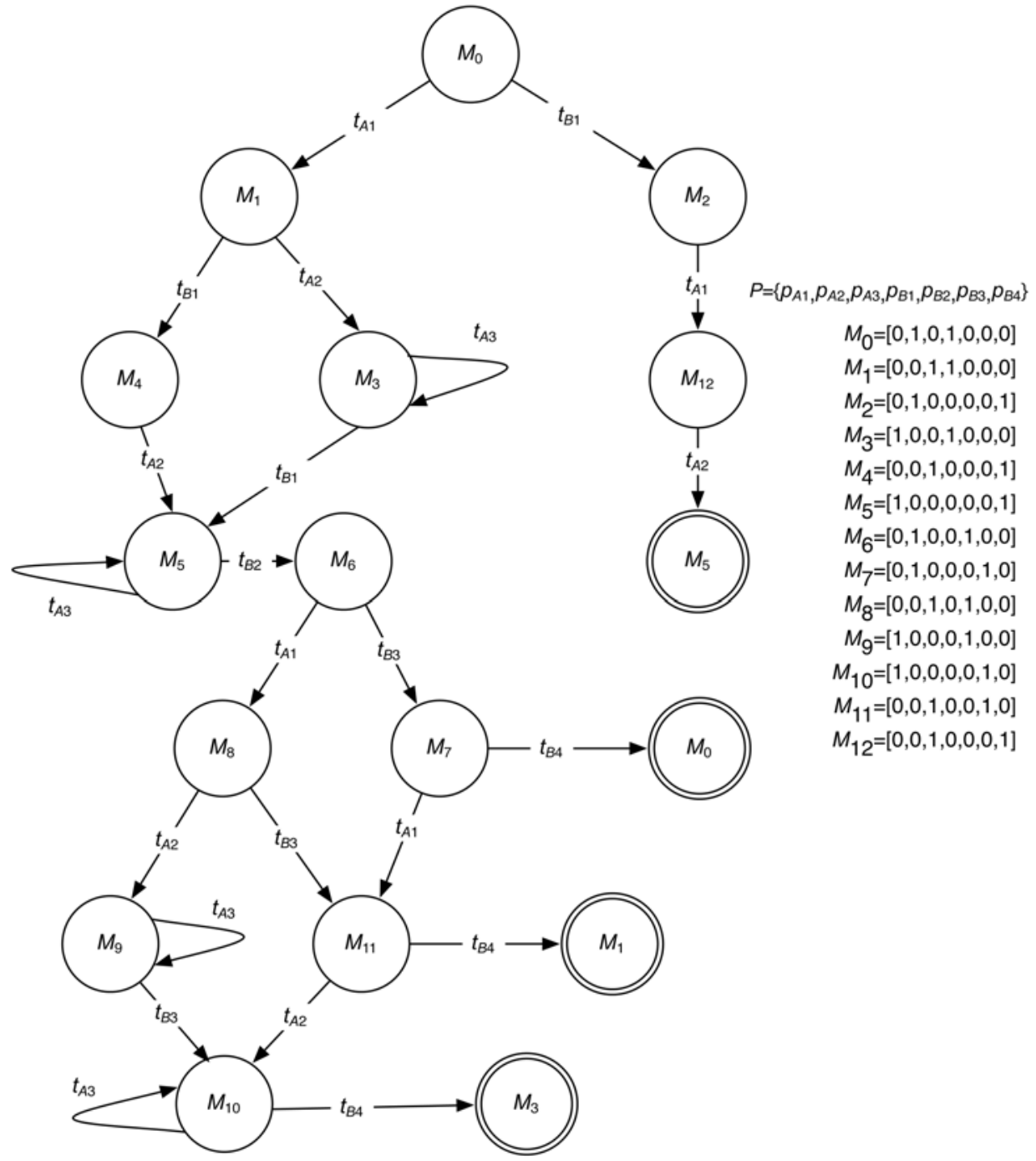

Fig. 11. Reachability graph of Petri net model N. 
The reachability graph shows the different markings that can be reached by firing different enabled transitions in the model. Every node in the graph represents a marking from the reachability set $R\left(M_{0}\right)$ of the initial marking. The arcs leaving each node indicate the transitions that are enabled, e.g., at marking $M_{6}$ transitions $t_{A 1}$ and $t_{B 3}$ are enabled. The initial marking $M_{0}=[0,1,0,1,0,0,0]$ represents the initial state of the system where a token is present in place $p_{A 2}$ and $p_{B 1}$, which in turn represents the initial state of both agents. It should be noted that the reachability graph is generated taking $M_{0}$ as a starting state, therefore, the assessment of properties using this graph will result in such properties being valid only when $M_{0}=[0,1,0,1,0,0,0]$.

It can be seen from this graph that at every marking of $R\left(M_{0}\right)$, there is always an enabled transition (every node in the graph has an outgoing arc). As a result the net is live when the initial marking (initial condition) is $M_{0}=[0,1,0,1,0,0,0]$.

The boundedness of the net when $M_{0}=[0,1,0,1,0,0,0]$ can also be observed from the reachability graph. The number of tokens present at a particular place is indicated for all the markings reachable from $M_{0}$, hence it is possible to observe the maximum number of tokens a place can have. The reachability graph of $M_{0}=[0,1,0,1,0,0,0]$ shows that the bound in the number of tokens for all the places in the net is one, therefore, the net is safe (1-bounded). Structural analysis is done using the incidence matrix (9) of the net and its invariants (10). It shows that the net is bounded and that the necessary condition for liveness is satisfied; this is a slightly weaker conclusion on "liveness" than when both necessary and sufficient conditions are met. The incidence matrix $A_{4}$ for net $N$ is a $m \times n$ matrix, where $m=|T|$ and $n$ $=|P|$. The row order of transitions is given by $T=\left\{t_{A 1}, t_{A 2}, t_{A 3}, t_{B 1}, t_{B 2}, t_{B 3}, t_{B 4}\right\}$ and the column order of places is $P=\left\{p_{A 1}, p_{A 2}, p_{A 3}, p_{B 1}, p_{B 2}, p_{B 3}, p_{B 4}\right\}$.

The incidence matrix gives an indication on the number of tokens gained for every place in the net as a result of a transition firing. The state equation (8) presented below, models the marking evolution as a function of firing transitions. It is defined as

$$
M_{f}=M_{0}+u A,
$$

were $u$ is a row vector of $m$ elements of nonnegative integers. The $i_{t h}$ element of $u$ represents the number of times transition $t_{i}$ is fired in order to reach $M_{f}$ from $M_{0} . M_{0}$ is the current marking of the net, and $M_{f}$ is the marking reached by firing the transitions indicated by $u$.

$$
\begin{aligned}
& A_{4}= {\left[\begin{array}{rrrrrrr}
0 & -1 & 1 & 0 & 0 & 0 & 0 \\
1 & 0 & -1 & 0 & 0 & 0 & 0 \\
0 & 0 & 0 & 0 & 0 & 0 & 0 \\
0 & 0 & 0 & -1 & 0 & 0 & 1 \\
-1 & 1 & 0 & 0 & 1 & 0 & -1 \\
0 & 0 & 0 & 0 & -1 & 1 & 0 \\
0 & 0 & 0 & 1 & 0 & -1 & 0
\end{array}\right] } \\
& \mathrm{T} \text {-invariants }=\left[\begin{array}{ll}
0 & 1 \\
0 & 1 \\
1 & 0 \\
0 & 1 \\
0 & 1 \\
0 & 1 \\
0 & 1
\end{array}\right] \text { and P-invariants }=\left[\begin{array}{ll}
1 & 0 \\
1 & 0 \\
1 & 0 \\
0 & 1 \\
0 & 1 \\
0 & 1 \\
0 & 1
\end{array}\right]
\end{aligned}
$$


The state equation for $N$ is $M_{f}=M_{0}+u A_{4}$. This equation can be used to find reachable markings as described by the reachability graph. Lets assume that a series of transition firings are executed in $N$ starting from $M_{0}=[0,1,0,1,0,0,0]$. These transitions are $t_{A 1}, t_{B 1}$, $t_{A 2}, t_{B 2}, t_{A 1}$ in this specific order, which renders $u=[2,1,0,1,1,0,0]$. Use the state equation to compute the current marking $M_{f}=[0,0,1,0,1,0,0]$. This is equivalent to marking $M_{8}$ from the reachability graph in figure 11 which was reached from $M_{0}$ after firing the same transitions in the same order.

The state equation is the basis for the structural analysis based on the P-invariants for boundedness and T-invariants for liveness (reachability). Let $x$ be a P-invariant of the net which by definition satisfies $A x=0$, e.g., $x_{1}=[1,1,1,0,0,0,0]^{T}$ is a P-invariant of $A_{4}$ from (10). Multiplying the state equation (8) by $x$ we get $M_{f} x=M_{0} x+u A x$. The last term of the right hand side of the equality is zero given that $x$ is a P-invariant. As a result $M_{f} x=M_{0} x$, which in turn indicates that the total number of tokens in the places for which the P-invariant is one (places $p_{A 1}, p_{A 2}$ and $p_{A 3}$, for P-invariant $x_{1}$ ) does not change; disregarding which transition or set of transitions was fired. This token conservation property is also independent from $M_{0}$, hence, it can be used to assess structural boundedness of such places.

For P-invariant $x_{1}, M_{f} x_{1}=M_{0} x_{1}$ implies that $M_{f}\left(p_{A 1}\right)+M_{f}\left(p_{A 2}\right)+M_{f}\left(p_{A 3}\right)=M_{0}\left(p_{A 1}\right)+M_{0}\left(p_{A 2}\right)$ $+M_{0}\left(p_{A 3}\right)$. This means that places $\left\{p_{A 1}, p_{A 2}, p_{A 3}\right\}$ are bounded by $k=M_{0}\left(p_{A 1}\right)+M_{0}\left(p_{A 2}\right)+$ $M_{0}\left(p_{A 3}\right)$. This result applies for every initial marking $M_{0}$. Furthermore, if the markings of these places are assumed to be finite, then it can be concluded that such places are $k$ bounded. Further analysis can be done if more knowledge of $M_{0}$ is assumed. For $M_{0}=[0,1,0,1,0,0$, $0], k=M_{0}\left(p_{A 1}\right)+M_{0}\left(p_{A 2}\right)+M_{0}\left(p_{A 3}\right)=1$, therefore these places are 1-bounded (safe).

The same methodology is used to make an assessment about the structural boundedness of the rest of the places of $N$. The second P-invariant vector in (10) is $x_{2}=[0,0,0,1,1,1,1]^{T}$. It can be concluded that places $\left\{p_{B 1}, p_{B 2}, p_{B 3}, p_{B 4}\right\}$ are bounded by $k=M_{0}\left(p_{B 1}\right)+M_{0}\left(p_{B 2}\right)+$ $M_{0}\left(p_{B 3}\right)+M_{0}\left(p_{B 4}\right)$ for every possible initial marking. Furthermore, since $M_{0}=[0,1,0,1,0,0,0]$, then $k=M_{0}\left(p_{B 1}\right)+M_{0}\left(p_{B 2}\right)+M_{0}\left(p_{B 3}\right)+M_{0}\left(p_{B 4}\right)=1$, therefore these places are 1-bounded (safe).

The structural boundedness by net invariants can also be assessed as described in theorem 1 . The basis for the theorem is the same as presented above, even though it is more restrictive since it is focused on assessing boundedness of the whole net. From theorem 1 it can only be concluded that the net is bounded, but doing a more detailed analysis of the situation allowed the conclusion that the net is safe. For cases where the model complexity is large, it could be difficult to find instances in which the whole net is bounded. In such cases, knowledge of the details of token conservation should be leveraged to provide boundedness assessment of submodels of the net.

Another use of the state equation (8) is in the solution of the reachability problem. The reachability problem consists of knowing whether marking $M_{f}$ can be reached by firing a sequence of transitions given that the net is currently on marking $M_{0}$. Basically, if $M_{f} \in R\left(M_{0}\right)$, then $u$ is a nonnegative integer solution to $M_{f}=M_{0}+u A$. Solution to the state equation is just a necessary condition for reachability. This is due to the fact that $u$ tells only which transitions are firing and how many firings per transition will result in marking $M_{f}$. It does not tell the order in which such transitions should be fired (sequence of transition firings), furthermore, it does not warranty that firing sequences consistent with $u$ will be feasible, given that there could exist transitions in the sequence that are not enabled in markings where they are expected to fire. 
The reachability problem is closely related to the liveness assessment. Structural liveness is difficult to prove based on the network invariants. As a matter of fact, only a necessary condition for structural liveness is available; which was described earlier in theorem 2. For net $N$, which is bounded and covered by T-invariants (8), the necessary condition for liveness is satisfied.

Further interpretation of the T-invariants, in addition to the fact that the state equation solution provides only a necessary condition for reachability, gives further insight on why a T-invariant coverage provides only a necessary condition for liveness. The T-invariant of a net is defined as $A^{T} y=0$, where $A$ is the incidence matrix. This equation is equivalent to $y^{T} A$ $=0$. Let $u=y^{T}$, then the state equation $M_{f}=M_{0}+y^{T} A$ becomes $M_{f}=M_{0}$. This means that firing the transitions described by the T-invariant does not change the marking of the network. Furthermore, by firing such transitions, the net returns to marking $M_{0}$. This property is key in the construction of theorem 2. In addition, the fact that only the set of transition firings is known, but not a feasible transition firing sequence, results in this theorem being a necessary condition only.

\section{Analysis of Petri net models from multi-agent systems}

This section presents the application of Petri net synthesis and reduction methods for the modeling and analysis of multi-agent systems using Petri nets. In particular, the use of synthesis and reduction methodologies for Petri net models of multi-agent systems with indirect interaction, and presents a simple example where the principles are demonstrated. This work builds on the methodology presented in section 3 which presents a systematic approach to building Petri net models of multi-agent systems with indirect interaction. In the examples presented in section 3 only a mutli-agent system consisting of two agents was studied and liveness and boundedness properties were assessed. The question now is how could these properties be proved for larger systems or for any multi-agent system in general that has indirect interaction. One way to do it will be to build the Petri net model for the entire multi-agent system and apply the structural or behavioral analysis approaches to the entire model in order to make an assessment. The drawback of this approach is the complexity involved in a) generating a detailed Petri net model for the complete system, b) generating reachability graphs for large Petri nets, and c) finding the invariants of a large incidence matrix since the null space of the matrix must be computed. Structural analysis is also restrictive since results must apply to every initial marking. An additional drawback could be the interpretation of such an assessment and its usefulness. Another approach is the divide and conquer approach where we can leverage Petri net synthesis and reduction methodologies for analysis to prove liveness and boundedness of the whole system. This is done either by proving the properties at a subsystem level and then building them up, or by reducing the complete model while preserving its properties until a model of manageable size is obtained to assess properties [16].

The synthesis and reduction approaches have been successfully applied to the analysis of Petri net models of manufacturing systems [17][18][16]. There is no evidence that these methodologies have been applied in the analysis of Petri net models of multi-agent systems. In general, the focus has been on obtaining Petri net models of specific multi-agent systems, but not in the analysis of such models. 


\subsection{Introduction to synthesis and reduction methods for Petri nets}

Petri net synthesis for analysis is the process of adding refinement to Petri net models in a manner in which certain properties like liveness and boundedness are preserved. The synthesis methodologies consist of starting with a simple model where certain properties hold, and then adding detail in the form of more places and transitions following rules that ensure that such properties will hold in the new more detailed model [17]. The synthesis and reduction methodologies can be described as: i) top-down, ii) bottom-up and iii) hybrid. The top-down approach starts with a high level model of the system under consideration and then adds stepwise refinements until the desired level of detail is achieved in the model [17][19][20]. The bottom-up approach also referred to as a reduction approach consists of joining Petri net sub-models that share places or transitions. This approach can also be described as transforming the detailed Petri net models using macroplaces and macrotransitions so as to obtain a smaller model where the desired properties can be proved [17][21][22].

Petri net transformations for analysis: Petri net transformations play a crucial role in the analysis and assessment of properties. Reduction and synthesis methodologies for analysis make use of transformations in order to facilitate the analysis. Such transformations are required to preserve system properties; which might vary depending on the focus of the analysis, e.g., liveness should be preserved if deadlock is to be assessed for the whole model. Transformations might involve places, transitions, or even sections of a Petri net. They range from removal of redundant transitions and places, to the exchange of a place or a transition by a submodel with higher level of detail. Property preserving transformations are usually bi-directional. If a type of transformation removes a redundant place preserving liveness, then the same transformation in reverse order can also be used the other way around to provide more detail preserving the liveness property.

Petri net model construction and analysis: In general, the differences between model assessment and model construction processes are well understood, since they are present for every type of modeling activity in engineering and science. The model analysis process starts when a model of a natural or man-made system is readily available, then analysis of key properties and behavior of such a model is performed. It is easy to visualize how reduction or even synthesis methodologies can be applied for this situation. On the other hand, model construction has subtle details that must be clarified in order to understand how the synthesis and reduction techniques can be applied to it.

For a model construction process for existing systems, either man-made or natural, the purpose is to build a model, often times a mathematical model which mimics the behavior of the existing system. There could be a multiple number of models ranging from different levels of detail to different methodologies. An example where different levels of detail are present is in dynamic systems, which could either be a lumped-parameter model (ordinary differential equations), a distributed parameter model (partial differential equation) or even a stochastic model (removing the assumption that some variables are deterministic). An example of different methodologies for modeling could be that of a manufacturing system; which can either be modeled as a queuing system, with discrete-event simulation, or even Petri nets. The selection of the level of detail and methodologies is often guided by the requirements of the problem at hand. 
For the Petri net case, synthesis methodologies can be used in the model construction process. One can start with a high level of abstraction model which presents certain key properties, additional levels of detail are then added by following property preserving transformations. A similar approach is to construct models of different sections of the system, then use synthesis procedures to join the different submodels in such a way that the properties of interest are preserved.

For a model construction of a system that does not already exist, like the control logic for an automated manufacturing facility, the purpose is to build a model that satisfies a series of conditions and properties. In such cases, models can be constructed taking desired properties into account during the construction process.

The concepts described above apply to the multi-agent system domain directly. There are cases where a multi-agent system is used to model an existing system, as in the case studies presented in previous sections. In other situations, like the control of the national airspace system, multi-agent system controllers have been proposed to carry out space management operations in a safe and efficient manner. In both cases, analysis methodologies should be employed to assess properties of the system. Such multi-agent systems models can be translated into the Petri net domain to assess deadlock or other properties. For a multi-agent system construction process, the development can start with a high-level model and then add more detail to the individual agents. Furthermore, Petri net synthesis concepts could be used to aid the design of interaction mechanisms among the agents in such a way that properties of the individual agents are preserved through the interaction.

On synthesis and reduction methodologies: The reduction approach presented in figure 4.1a shows how starting from a high fidelity model with high-level of detail, the model is reduced to a size manageable to perform behavioral or structural analysis. The model $N_{a 1}$ is a high-level of detail model used as a starting point, transformation $T_{a 1}$ reduces the model in complexity resulting in a new model $N_{a 2}$. Transformation $T_{a 1}$ should be a property preserving transformation so that $N_{a 2}$ preserves the properties of interest from $N_{a 1}$. For example, if liveness and boundedness properties are to be assessed for $N_{a 1}$, then transformations $T_{a 1}$ and $T_{a 2}$ must preserve these properties. The final model $N_{a 3}$ will have lower complexity than the high fidelity model. Analysis of $N_{a 3}$ is now easier or even possible if the complexity of $N_{a 1}$ is such that analysis is intractable. The following are situations when the reduction approach is helpful.

- When a high fidelity model is too large to assess behavioral properties of the complete model. For this situation, the number of markings in $R\left(M_{0}\right)$ is too large and the construction of the reachability graph is cumbersome even with the aid of computational tools.

- When there is more interest in the behavior of a section of the model and how it interacts with the rest of the model. In this situation, the rest of the net could be reduced as much as possible to facilitate the analysis of the section of interest.

The synthesis approach is also depicted in figure 4.1b. Synthesis methods focus on the composition of models from submodels. Two submodels can be joined to form a larger one or a place or transition can be exchanged for a submodel with higher level of detail. Similar to the reduction approach, these transformations should also be property preserving. Assuming that transformations $T_{b 1}$ and $T_{b 2}$ preserve properties like liveness and 
boundedness, then model $N_{b 3}$ will be live and bounded if model $N_{b 1}$ is live and bounded. This allows one to perform the analysis in the model of smaller complexity and then extrapolate those results for the larger model.

\subsection{Literature review}

The work by Suzuki in [23] and Suzuki and Murata in [20] presents a methodology for expanding and reducing Petri nets to a desired level of detail. This methodology consists of stepwise refinements of transitions and places, as well as abstraction of subnets into a single

a) Reduction approach

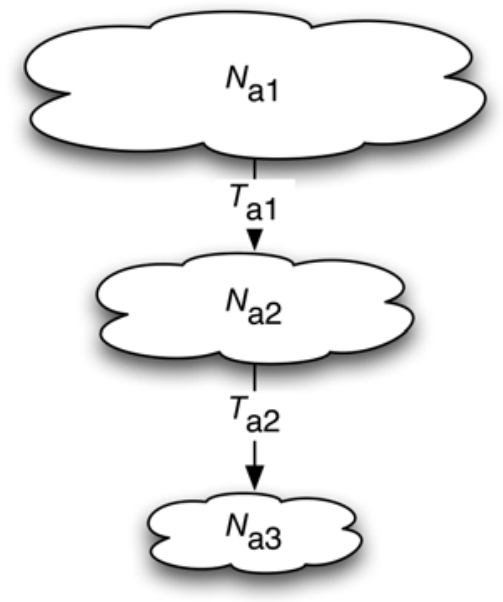

b) Synthesis approach

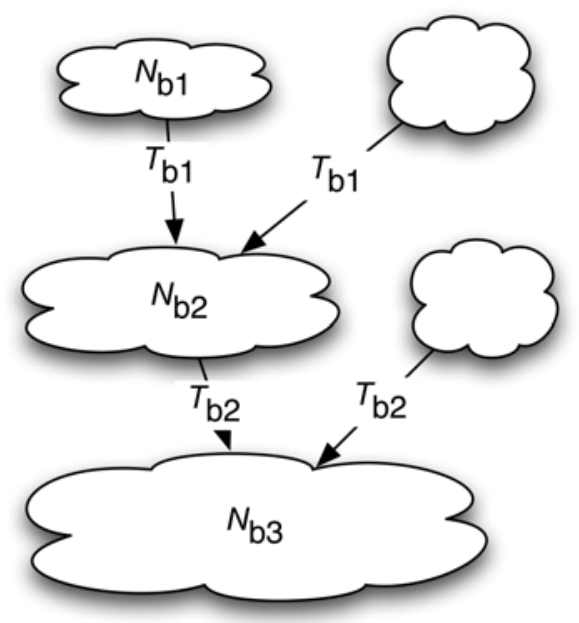

Fig. 12. Synthesis and reduction of Petri net models.

transition. The transformations presented preserve liveness and boundedness. Reduction is presented as a substitution of a subnet by a single transition, hence generating a new model with higher level of abstraction.

Synthesis is approached by way of stepwise refinements. Datta and Ghosh in [24] present a top-down approach to analysis of regular Petri nets. Regular Petri nets are live and bounded by construction. The properties considered to be preserved by the transformations are liveness and boundedness. The work by Lee and Favrel in [22] presents a hierarchical (bottom-up) approach to analysis. This consists of a hierarchical reduction and decomposition by transforming subnets into macroplaces or macrotransitions. Liveness and boundedness are preserved by these transformations. The work by Berthelot in [25] presents a series of transformations to provide reduction and synthesis. The properties considered include liveness, boundedness, safety, covering of P-invariants, return to home state and others. Place transformations and fusion of transitions are presented to provide reduction. Synthesis is considered by addition of submodels. Lee et al. in [21] present a reduction methodology for generalized Petri nets. In generalized Petri nets, multiple arcs are allowed between transitions and places, as a result, the weights of the arcs can be larger than one. The purpose of the methodology is to reduce the state-space of the net to a manageable size based on the structure of the net. Liveness, boundedness and proper termination properties 
are preserved by reducing subnets into macroplaces or macrotransitions. The work by Jeng and DiCesare in [26] presents a review of synthesis and reduction methods. Examples of applications in the manufacturing domain are presented as well. Zhou et al. in [18] present a hybrid methodology for synthesis of Petri net models of automated manufacturing systems. In this work, modeling details are included as increments by refining places. Reversibility, boundedness and liveness are preserved by this methodology. The work by Jeng and DiCesare [27] presents a review of reduction and synthesis technologies and their applications to manufacturing. Methodologies for synthesis are presented by considering merging of places from different subnets, and refinement of transitions. The properties preserved depend on the transformations applied. Synthesis and analysis of flexible manufacturing systems is presented in Zhou et al. [16]. In this work, the synthesis process is used for the model construction process. Petri net design approaches and the modeling process are considered, as well as the analysis of the system. Refinement is used to design a system that conforms to certain desired properties like liveness, boundedness and reversibility. The work by Jeng in [28] presents a methodology for synthesis of flexible manufacturing systems. A bottom-up approach is used to construct models and interactions among submodels is investigated. This is an application of reduction and refinement for the construction of Petri net models of flexible manufacturing systems.

\subsection{Analysis of Petri net model $\boldsymbol{N}$ using reduction techniques}

The Petri net model $N$ from the example (figure 13) in the previous section is presented here to illustrate how reduction techniques are used for the assessment of liveness and boundedness. From the results presented in the previous section we learned that this Petri net model is live and bounded for the initial marking $M_{0}$ (which is indicated in figure 13). The Petri net is also structurally bounded given that the number of places in the model must be finite in the initial condition. For structural liveness, only the necessary condition is satisfied. From the individual agent submodels (figure 6 and figure 8 ) it can be concluded that only the model for agent $\mathrm{B}$ is live and bounded.

The objective here is to prove that the model is live and bounded. In order to do so, it is assumed that it is not known in advance that the model is live and bounded. By applying the reduction techniques, the model will be reduced in size until the liveness and boundedness properties are evident from the reduced model.

Transformations: The transformations considered for this example are presented in [17]. Basically, two transition transformations fusion and pre-fusion will be used to reduce the model. These transformations preserve liveness and boundedness, and they are intended to reduce the number of reachable markings of the net; making easier the analysis of the reduced model.

The fusion transformation combines two transitions $t_{i}$ and $t_{j}$ that are separated by a single place $p_{k}$. Place $p_{k}$ must be the only output place of transition $t_{i}$ and it is also an input place of transition $t_{j}$. The transformation removes place $p_{k}$ and combines the transitions into a single transition $t_{i j}$, which will have the same input places as $t_{i}$ and the same output places as $t_{j}$. This transformation preserves liveness and boundedness [17].

The pre-fusion is a liveness and boundedness preserving transformation that combines two transitions $t_{i}$ and $t_{j}$ that are separated by a single place $p_{k}$. Place $p_{k}$ must be the only output 
place of transition $t_{i}$ and it is also an input place of transition $t_{j}$. In addition, transition $t_{j}$ can have additional input places besides $p_{k}$. The transformation removes place $p_{k}$ and combines the transitions into a single transition $t_{i j}$, which will have the same input places as $t_{i}$ and the same input and output places as $t_{j}[17]$.

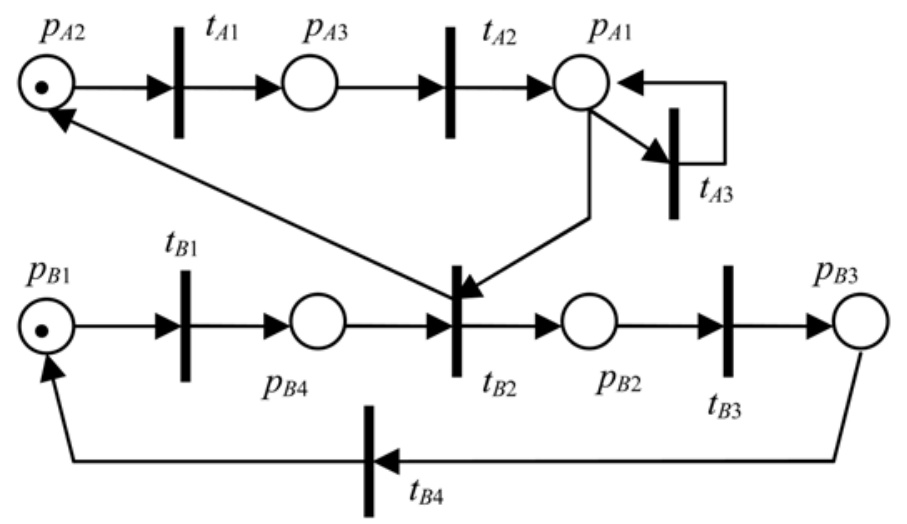

Fig. 13. Petri net model $N$.

Reduction procedure: The Petri net model $N$ is presented in figure 14 . In this figure, a series of fusion transformations are indicated. Transitions $t_{A 1}$ and $t_{A 2}$ will be fused, as a result, place $p_{A 3}$ will be removed resulting in the model in figure 15a. Fusion of transitions is applied twice in the bottom part of the model. First transitions $t_{B 1}$ and $t_{B 4}$ are fused. The resulting transition is then fused with transition $t_{B 3}$ to form transition $t_{B 134}$ in figure 15a. In addition, transition $t_{A 3}$ was removed since it does not change the marking when it fires.

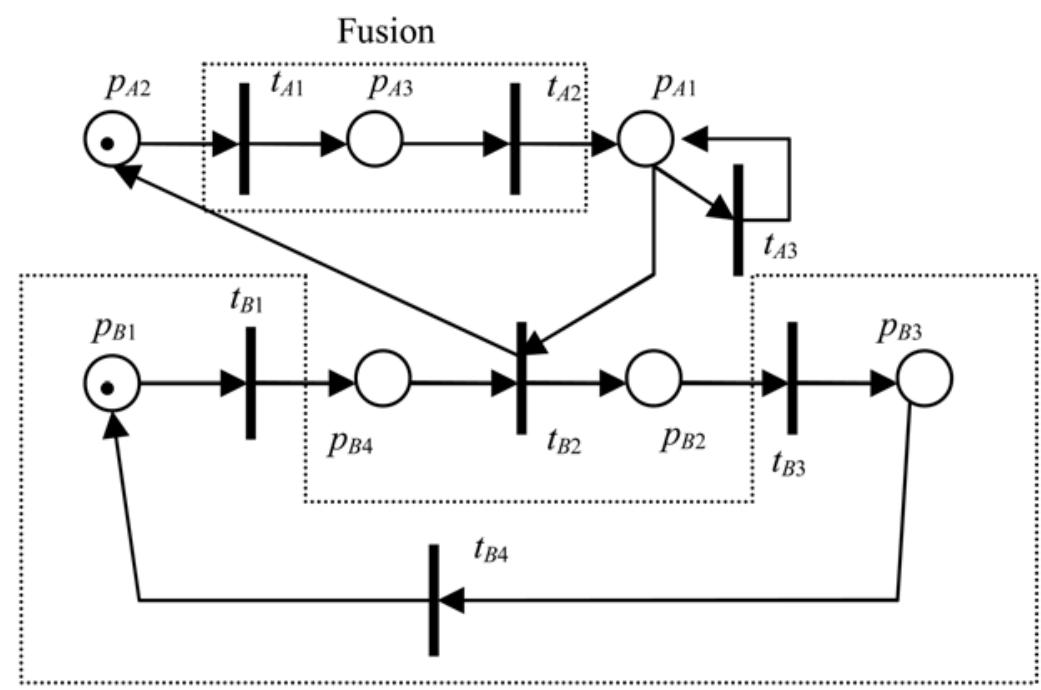

Fusion

Fig. 14. Fusion transformations in Petri net model N. 
The final model is presented in figure $15 \mathrm{~b}$. It is obtained after applying the pre-fusion transformation to the model in figure 15a.

The pre-fusion transformation will remove place $p_{B 4}$ and combine transition $t_{B 2}$ with transition $t_{B 134}$. The resulting model is sufficiently simple to perform the assessment of liveness and boundedness by inspection. The size of the reachability set is two since there are only two possible markings in the model. The maximum number of tokens in all the places is one, as a result the net is 1-bounded (safe). It is also evident that there is always an enabled transition, as a result the model is live.

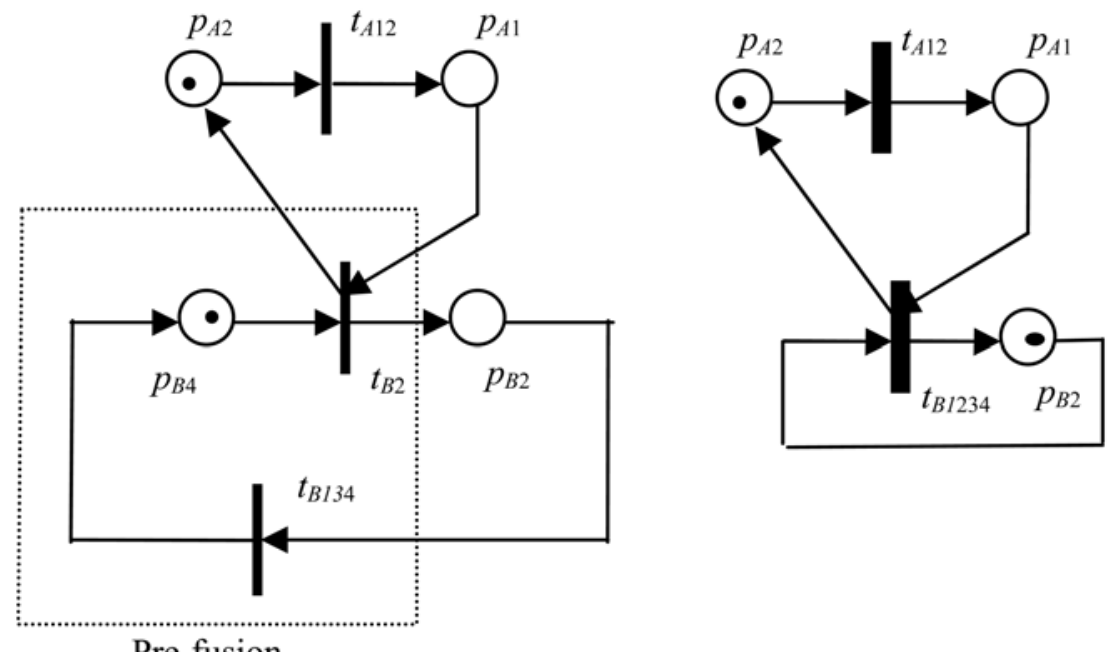

Pre-fusion

Fig. 15. Reduced Petri net model N.

Since all the transformations applied preserve liveness and boundedness, the fact that the reduced model is live and bounded implies that the original Petri net model (figure 13) is also live and bounded.

\section{Discussion}

This chapter presented a methodology for the modeling and analysis of multi-agent systems using Petri nets. Multi-agent systems with indirect interaction were considered as a starting point for the development of Petri net based tools for multi-agent systems.

The interaction framework considered was the simplest means of interaction among agents and referred to in this work as indirect interaction. In the indirect interaction framework agents can interact with each other by changing each other's environment. At the interaction level, it is natural to regard a multi-agent system as a discrete-event system, and by focusing at this level, this work presents a methodology to assess how indirect interactions will work with respect to the discrete-event system properties of the multiagent system. Petri nets have been used in several applications of discrete-event dynamic systems. In this work, multi-agent systems were regarded as a discrete event-system and modeled using the Petri net methodology. Thus, properties of Petri nets were analyzed and related to multi-agent 
systems. The liveness and boundedness properties in the Petri net domain are important for multi-agent systems as they relate to deadlock avoidance. Furthermore, properties from the Petri net domain could be related to characteristics of the communication and interaction mechanism of the multi-agent system. The abstract architecture of intelligent agents is considered as a preliminary step of the Petri net model construction process. The methodology proposed in this work consists of describing a multi-agent system with the abstract architecture for intelligent agents, and then constructing a Petri net model systematically from the abstract architecture description of the system. Different scenarios were considered in order to indicate how the Petri net analysis methodologies can be used to assess key system properties, showing that there is a relationship between multi-agent systems with indirect interaction and Petri nets. The mapping of multi-agent systems models with the abstract architecture into Petri net models captured the discrete-event dynamics of the systems under consideration. This allowed the assessment of properties like deadlock avoidance in a multi-agent system, which can be assessed systematically from the Petri net model.

In general, the results presented in this work show the potential for using Petri nets to assess key properties of multi-agent systems. In addition, they provide the foundation to further investigate the application of Petri net methodologies for the modeling, analysis and design of multi-agent systems.

\section{References}

[1] T. Murata, P. C. Nelson, and J. Yim, "A predicate-transition net model for multiple agent planning," Inf. Sci., vol. 57-58, pp. 361-384, 1991.

[2] D. Xu, R. Volz, T. Ioerger, and J. Yen, "Modeling and verifying multi-agent behaviors using predicate/transition nets," in SEKE '02: Proceedings of the 14th international conference on Software engineering and knowledge engineering, (New York, NY, USA), pp. 193-200, ACM Press, 2002.

[3] H. J. Ahn and S. J. Park, "Modeling of a multi-agent system for coordination of supply chains with complexity and uncertainty," in Intelligent Agents and Multi-Agent Systems (J. Lee and M. Barley, eds.), vol. 2891 of Lecture Notes in Computer Science, pp. 13-24, 6th Pacific Rim International Workshop on Multi-Agents, PRIMA 2003 Seoul, Korea, Springer-Verlag Berlin Heidelberg, November 2003.

[4] P. Leitão, A. W. Colombo, and F. Restivo, "An approach to the formal specification of holonic control systems," in Holonic and Multi-Agent Systems for Manufacturing (V. Marík, D. McFarlane, and P. Valckenaers, eds.), vol. 2744 of Lecture Notes in Computer Science, pp. 59-70, First International Conference on Industrial Applications of Holonic and Multi-Agent Systems, HoloMAS 2003 Prague, Czech Republic, September 1-3, 2003, Springer Berlin / Heidelberg, 2004.

[5] F.-S. Hsieh, "Model and control holonic manufacturing systems based on fusion of contract nets and Petri nets," Automatica, vol. 40, no. 1, pp. 51-57, 2004.

[6] M. R. Lyu, X. Chen, and T. Y. Wong, "Design and evaluation of a fault-tolerant mobileagent system," Intelligent Systems, vol. 19, no. 5, pp. 32-38, 2004.

[7] A. W. Krings, "Agent survivability: An application for strong and weak chain constrained scheduling," in HICSS '04: Proceedings of the Proceedings of the 37th 
Annual Hawaii International Conference on System Sciences (HICSS'04) - Track 9, (Washington, DC, USA), p. 90297.1, IEEE Computer Society, 2004.

[8] T. Murata, "Petri nets: Properties, analysis and applications," Proceedings of the IEEE, vol. 77, pp. 541-580, April 1989.

[9] T. Agerwala, "Putting Petri nets to work," Computer, vol. 12, pp. 85- 94, December 1979.

[10]C. G. Cassandras, Discrete Event Systems, Modeling and Performance Analysis. Aksen Associates Incorporated, 1993.

[11] A. A. Desrochers, "Performance analysis using Petri nets, "Journal of Intelligent and Robotic Systems, vol. 6, pp. 65-79, August 1992.

[12] J. L. Peterson, Petri net theory and the modeling of systems. Prentice Hall, 1981.

[13] W. Reisig, Petri nets, An Introduction, vol. 4 of EATCS: Monographs on Theoretical Computer Science. Springer-Verlag, 1985.

[14] M. J. Wooldridge, Introduction to Multiagent Systems. John Wiley \& Sons, Inc., 2001.

[15] K. P. Sycara, "Multiagent systems," AI Magazine, pp. 79-92, 1998.

[16] M. Zhou, K. McDermott, and P. A. Patel, "Petri net synthesis and analysis of a flexible manufacturing system cell," IEEE Transactions on Systems, Man, and Cybernetics, vol. 23, pp. 523-531, March 1993.

[17] A. A. Desrochers and R. Y. Al-Jaar, Applications of Petri Nets in Manufacturing Systems: Modeling, Control, and Performance Analysis. IEEE Press, 1995.

[18] M. Zhou, F. DiCesare, and A. A. Desrochers, "A hybrid methodology for synthesis of Petri net models for manufacturing systems," IEEE Transactions on Robotics and Automation, vol. 8, no. 3, pp. 350-361, 1992.

[19] R. Valette, "Analysis of Petri nets by stepwise refinements," Journal of Computer and System Sciences, vol. 18, no. 1, pp. 35-46, 1979.

[20] I. Suzuki and T. Murata, "A method for stepwise refinement and abstraction of Petri nets," Journal of Computer and System Sciences, vol. 27, no. 1, pp. 51-76, 1983.

[21] K. H. Lee, J. Favrel, and P. Baptiste, "Generalized Petri net reduction method.," IEEE Transactions on Systems Man and Cybernetics, vol. 17, no. 2, pp. 297-303, 1987.

[22] K. H. Lee and J. Favrel, "Hierarchical reduction method for analysis and decomposition of Petri nets," IEEE Transactions on Systems, Man, and Cybernetics, vol. 15, no. 2, pp. 272-280, 1985.

[23] I. Suzuki and T. Murata, "Stepwise refinements of transitions and places," in Selected Papers from the First and the Second European Workshop on Application and Theory of Petri Nets, (London, UK), pp. 136-141, Springer-Verlag, 1982.

[24] A. Datta and S. Gosh, "Synthesis of a class of deadlock-free Petri nets," Journal of the Association for Computing Machinery, vol. 31, pp. 486-506, July 1984.

[25] G. Berthelot, "Checking properties of nets using transformations," in Advances in Petri Nets 1985, covers the 6th European Workshop on Applications and Theory in Petri Nets-selected papers, pp. 19-40, Springer-Verlag, 1986.

[26] M. D. Jeng and F. DiCesare, "A review of synthesis techniques for Petri nets," in Rensselaer's Second International Conference on Computer Integrated Manufacturing, pp. 348-355, 1990. 
[27] M. D. Jeng and F. DiCesare, "A review of synthesis techniques for Petri nets with applications to automated manufacturing systems," IEEE Transactions on Systems, Man and Cybernetics, vol. 23, no. 1, pp. 301-312, 1993.

[28] M. D. Jeng, "A Petri net synthesis theory for modeling flexible manufacturing systems," IEEE Transactions on Systems, Man, and Cybernetics, Part B: Cybernetics, vol. 27, pp. 169-183, April 1997. 


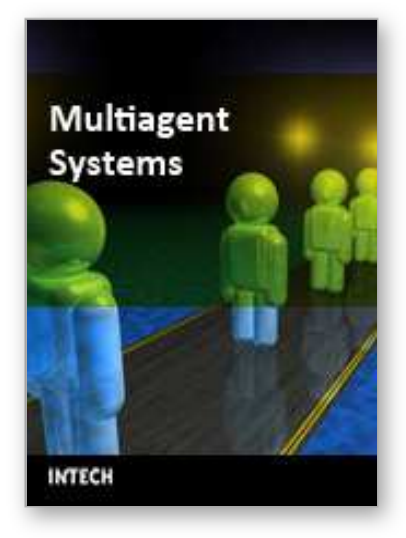

\author{
Multiagent Systems \\ Edited by Salman Ahmed and Mohd Noh Karsiti
}

ISBN 978-3-902613-51-6

Hard cover, 426 pages

Publisher I-Tech Education and Publishing

Published online 01, January, 2009

Published in print edition January, 2009

Multi agent systems involve a team of agents working together socially to accomplish a task. An agent can be social in many ways. One is when an agent helps others in solving complex problems. The field of multi agent systems investigates the process underlying distributed problem solving and designs some protocols and mechanisms involved in this process. This book presents an overview of some of the research issues in the field of multi agents. It is a presentation of a combination of different research issues which are pursued by researchers in the domain of multi agent systems as they are one of the best ways to understand and model human societies and behaviours. In fact, such systems are the systems of the future.

\title{
How to reference
}

In order to correctly reference this scholarly work, feel free to copy and paste the following:

Jose R. Celaya and Alan A. Desrochers (2009). Modeling and Analysis Methods for Multi-Agent Systems, Multiagent Systems, Salman Ahmed and Mohd Noh Karsiti (Ed.), ISBN: 978-3-902613-51-6, InTech, Available from: http://www.intechopen.com/books/multiagent_systems/modeling_and_analysis_methods_for_multiagent_systems

\section{INTECH}

open science | open minds

\section{InTech Europe}

University Campus STeP Ri

Slavka Krautzeka 83/A

51000 Rijeka, Croatia

Phone: +385 (51) 770447

Fax: +385 (51) 686166

www.intechopen.com

\section{InTech China}

Unit 405, Office Block, Hotel Equatorial Shanghai

No.65, Yan An Road (West), Shanghai, 200040, China

中国上海市延安西路65号上海国际贵都大饭店办公楼 405 单元

Phone: +86-21-62489820

Fax: $+86-21-62489821$ 
(C) 2009 The Author(s). Licensee IntechOpen. This chapter is distributed under the terms of the Creative Commons Attribution-NonCommercialShareAlike-3.0 License, which permits use, distribution and reproduction for non-commercial purposes, provided the original is properly cited and derivative works building on this content are distributed under the same license. 